\title{
On microphysical processes of noctilucent clouds (NLC): observations and modeling of mean and width of the particle size-distribution
}

\author{
G. Baumgarten, J. Fiedler, and M. Rapp \\ Leibniz-Institute of Atmospheric Physics at the Rostock University, 18225 Kühlungsborn, Germany
}

Received: 11 December 2009 - Published in Atmos. Chem. Phys. Discuss.: 9 February 2010

Revised: 18 May 2010 - Accepted: 7 July 2010 - Published: 21 July 2010

\begin{abstract}
Noctilucent clouds (NLC) in the polar summer mesopause region have been observed in Norway $\left(69^{\circ} \mathrm{N}\right.$, $16^{\circ}$ E) between 1998 and 2009 by 3-color lidar technique. Assuming a mono-modal Gaussian size distribution we deduce mean and width of the particle sizes throughout the clouds. We observe a quasi linear relationship between distribution width and mean of the particle size at the top of the clouds and a deviation from this behavior for particle sizes larger than $40 \mathrm{~nm}$, most often in the lower part of the layer. The vertically integrated particle properties show that $65 \%$ of the data follows the linear relationship with a slope of $0.42 \pm 0.02$ for mean particle sizes up to $40 \mathrm{~nm}$. For the vertically resolved particle properties $(\Delta z=0.15 \mathrm{~km})$ the slope is comparable and about $0.39 \pm 0.03$. For particles larger than $40 \mathrm{~nm}$ the distribution width becomes nearly independent of particle size and even decreases in the lower part of the layer. We compare our observations to microphysical modeling of noctilucent clouds and find that the distribution width depends on turbulence, the time that turbulence can act (cloud age), and the sampling volume/time (atmospheric variability). The model results nicely reproduce the measurements and show that the observed slope can be explained by eddy diffusion profiles as observed from rocket measurements.
\end{abstract}

\section{Introduction}

Noctilucent clouds (NLC; also called polar mesospheric clouds, or PMC, when seen from space) are an intriguing optical twilight phenomenon which can be observed throughout the summer months, most often at latitudes poleward of $50^{\circ}$ (e.g. Thomas, 1984; Gadsden and Schröder, 1989). NLC consist of water ice particles which form in the extremely cold and dry environment of the polar summer mesopause region (Hervig et al., 2001). This region is characterized by mean temperatures being as low as $\sim 130 \mathrm{~K}$ and by water vapor mixing ratios of just a few parts per million by volume (ppmv) (e.g Lübken, 1999; Seele and Hartogh, 1999). Temperature and water vapor in the polar summer mesopause region are driven by dynamical processes from global to local scales. Both scales are connected with gravity waves and wave breakdown, which can be seen directly in NLC displays (Witt, 1962; Fritts et al., 1993). Existing just at the edge of feasibility, NLC properties are extremely sensitive toward changes of their environment (e.g., temperature, water vapor, or dynamical parameters like wave activity). We report on observations of particle properties by multi-color lidar performed in Northern Norway (Baumgarten et al., 2008). We investigate in detail the mean and the width of the size distribution and compare the results to microphysical modeling of NLC to identify the processes affecting especially the distribution width. Besides the microphysical aspects these observations are important for the interpretation of other instruments sounding the particle size of NLC. For most instruments the particle size is retrieved under the assumption of a predefined and constant distribution width (e.g. Bailey et al., 2009; Robert et al., 2009). From our measurements we show that this assumption needs to be revisited.

In the following section we will briefly describe the observation method, including the data analysis procedure, and the microphysical model focused on the sensitivity study used for interpretation of the observations. In Sect. 3 we will present the observations and in Sect. 4 we will discuss the observations as well as the underlying microphysical processes.

Correspondence to: G. Baumgarten

(baumgarten@iap-kborn.de)

Published by Copernicus Publications on behalf of the European Geosciences Union. 


\section{Instrument, method and model}

\subsection{Lidar}

Lidar measurements of NLC particle properties were performed with the ALOMAR RMR-lidar in Northern Norway $\left(69^{\circ} \mathrm{N}, 16^{\circ} \mathrm{E}\right)$. Throughout the NLC season (1 June to 15 August) from 1998 to 2009 the lidar was operated whenever permitted by the weather. Laser pulses at three widely separated wavelengths $(355 \mathrm{~nm}, 532 \mathrm{~nm}, 1064 \mathrm{~nm})$ are emitted, scattered back by air molecules and particles in the atmosphere and collected by telescopes with a diameter of $1.8 \mathrm{~m}$. The received light is recorded by single photon counting detectors. After separation of particle and molecular signal, the particle properties are calculated by comparison to modeled optical particle signals (Baumgarten et al., 2007). The method is appropriate for the analysis of a mono-modal size distribution consisting of non-spherical particles, where the radius is that of a volume-equivalent sphere. Throughout this manuscript we use results for cylindrical particles and an assumed Gaussian-shaped size distribution (Berger and von Zahn, 2002; Rapp and Thomas, 2006). We analyze the particle properties throughout the NLC layer where the NLC signal is larger than twice the measurement uncertainty:

$\beta_{532 \mathrm{~nm}, \mathrm{NLC}}(z)>2 \times \Delta \beta_{532 \mathrm{~nm}, \mathrm{NLC}}(z)$, with $\beta$ being the backscatter coefficient and $\Delta \beta$ the corresponding measurement uncertainty. The measurements of NLC are analyzed for particle sizes only when in addition the measurement errors of the color ratios $C R_{\lambda, \mathrm{NLC}}=\beta_{\lambda, \mathrm{NLC}} / \beta_{532 \mathrm{~nm}, \mathrm{NLC}}$ are small. In detail: $\Delta C R_{1064 \mathrm{~nm}, \mathrm{NLC}}(z)<0.08$ and

$\Delta C R_{355 \mathrm{~nm}, \mathrm{NLC}}(z)<1.0$. These limits were found to give a good compromise of the precision of the single measurement and the number of measurements analyzed.

To enhance the signal to noise ratio, the data throughout the layer are processed in different ways: Minimal averaging using a sliding binomial filter with FWHM $=475 \mathrm{~m}$ and $150 \mathrm{~m}$ sampling (method 1). Segmentation of the layer into top, peak and bottom part (method 2). Usage of the vertically integrated signal (method 3). For the segmentation of the layer (method 2) we use the following algorithm: The peak range is defined to be the altitude range above and below the peak backscattering $\left(\beta_{\max } \equiv \beta_{532 \mathrm{~nm}, \mathrm{NLC}}\left(z_{\max }\right)\right)$ where $\beta_{532 \mathrm{~nm}, \mathrm{NLC}}(z)>0.7 \times \beta_{\max }$. The top and the bottom parts of the layer are calculated by summing up the significant backscattering above or below the peak range. We regard the NLC signal to be significant when it is larger than twice the measurement uncertainty. Further details on the analysis of the vertical structure throughout the NLC layer can be found in Baumgarten and Fiedler (2008). While method 1 shows the particle properties at the highest possible resolution, method 3 should be more comparable to other sounding methods (e.g. nadir viewing satellite instruments). Method 2 allows to study different aspects of the cloud microphysics. As the cloud particles fall through the atmosphere while they grow, younger particles should be found above the peak of the layer, while mature particles are expected at the layer bottom.

\subsection{CARMA}

The community aerosol and radiation model for atmospheres (CARMA) is a microphysical model developed over the past 30 years, and has been applied to a wide variety of problems ranging from cloud physics to aerosols. The model originates from a stratospheric aerosol code developed by Turco et al. (1979) and Toon et al. (1979). CARMA was first applied to the physics of mesospheric ice particles by Turco et al. (1982), and then further developed by several authors (e.g. Jensen and Thomas, 1994; Rapp et al., 2002). For the current study, we use results from a large number of simulations using a one-dimensional version of CARMA which have been described in detail in Rapp and Thomas (2006); Rapp et al. (2007).

\section{Observations}

The ALOMAR RMR-lidar has been operated for $3972 \mathrm{~h}$ during the NLC seasons from 1998 to 2009 whereof about 1680 hours contain signatures of NLC. A detailed description of the NLC dataset and the mean particle properties can be found in Fiedler et al. (2009) and Baumgarten et al. (2008) respectively. The lidar is designed as a twin system and measurements can be performed at two different locations separated by about $40 \mathrm{~km}$ at NLC altitude (Baumgarten et al., 2002). For the particle soundings we treat these as independent observations. This data set was analyzed with a temporal resolution of $14 \mathrm{~min}$. In total this results in 22820 soundings that could be analyzed for particle properties. Due to instrument developments the number of particle size soundings has increased since 2005. About $92 \%$ of the size measurements were performed in the years 2006 to 2009.

In Fig. 1 we show the retrieved width $(s)$ and the mean $(r)$ of the particle size distribution, color-coded as two dimensional probability distribution relative to the maximum. The figure shows results for the total dataset sampled with the highest possible resolution (method 1). The ensemble of particles with $r=31 \mathrm{~nm}$ and $s=13 \mathrm{~nm}$ occur most often and hence show the highest probability density. The mean distribution width for different particle sizes, calculated by binning the sizes in $2.5 \mathrm{~nm}$ steps, is also shown in the figure. We find two different relationships between distribution width and mean radius: For particle sizes below $40 \mathrm{~nm}$ width and radius are strongly correlated, while for larger particle sizes the distribution width is nearly constant. To give a simple function for this behavior, weighted linear regressions were performed for these two particle size ranges.

The weight of the regression is defined by the measurement uncertainty, in case of the size-binned mean (blackred curve in Fig. 1) we use the statistical error of the mean. 
Table 1. Particle properties and slope $(S)$ of the fit for different analysis methods/layer parts. I: Vertical integral, H: Highest resolution, T: NLC top, P: NLC peak, B: NLC bottom. The probability of finding particle soundings close to the fitted slope $( \pm 25 \%)$ is listed in column onfit. The probability for finding particle ensembles where the width is smaller than expected from the fit is listed in column below. $\langle r\rangle$ and $\langle s\rangle$ are the mean radius and mean width, respectively. $s_{35 \mathrm{~nm}}$ and $s_{50 \mathrm{~nm}}$ give the width at $r=35 \mathrm{~nm}$ and $r=50 \mathrm{~nm}$, respectively. The width of the layer is shown in column $\Delta z$ and the number of measurements is found in the last column. Cloud type "all" includes all clouds observed, while "statistic" denotes clouds with $\beta_{\max }>4 \times 10^{-10} \mathrm{~m}^{-1} \mathrm{sr}^{-1}$ used for statistical studies (e.g. Baumgarten et al., 2008).

\begin{tabular}{ccccccccccccr}
\hline Typ & Method & Cloud & $\begin{array}{c}S_{<40 \mathrm{~nm}} \\
{[1]}\end{array}$ & $\begin{array}{c}S_{\geq 40 \mathrm{~nm}} \\
{[1]}\end{array}$ & $\begin{array}{c}\text { onfit } \\
{[\%]}\end{array}$ & $\begin{array}{c}\text { below } \\
{[\%]}\end{array}$ & $\begin{array}{c}\langle\mathrm{nm}\rangle \\
{[\mathrm{nm}]}\end{array}$ & {$[\mathrm{nm}]$} & $\begin{array}{r}s_{35 \mathrm{~nm}} \\
{[\mathrm{~nm}]}\end{array}$ & $\begin{array}{c}s_{50 \mathrm{~nm}} \\
{[\mathrm{~nm}]}\end{array}$ & $\begin{array}{c}\Delta z \\
{[\mathrm{~km}]}\end{array}$ & Number \\
\hline I & 3 & all & $0.42 \pm 0.02$ & $-0.08 \pm 0.02$ & 65.4 & 31.1 & 42.8 & 13.8 & $14.3 \pm 0.9$ & $15.5 \pm 0.1$ & 2.13 & 1909 \\
H & 1 & all & $0.39 \pm 0.03$ & $0.003 \pm 0.008$ & 70.1 & 28.5 & 45.6 & 14.0 & $13.3 \pm 0.1$ & $14.7 \pm 0.2$ & 0.15 & 22820 \\
$\mathrm{~T}$ & 2 & all & $0.41 \pm 0.02$ & $-0.05 \pm 0.02$ & 78.2 & 19.9 & 37.3 & 12.8 & $13.7 \pm 0.9$ & $14.8 \pm 0.1$ & 0.92 & 865 \\
P & 2 & all & $0.44 \pm 0.02$ & $-0.08 \pm 0.02$ & 61.2 & 37.2 & 44.8 & 13.9 & $14.1 \pm 0.9$ & $15.4 \pm 0.1$ & 0.76 & 1928 \\
B & 2 & all & $0.44 \pm 0.03$ & $-0.08 \pm 0.02$ & 49.1 & 49.2 & 55.0 & 15.0 & $14.1 \pm 0.1$ & $16.8 \pm 0.2$ & 0.65 & 898 \\
I & 3 & statistic & $0.46 \pm 0.03$ & $-0.07 \pm 0.02$ & 57.0 & 41.5 & 43.9 & 14.1 & $14.7 \pm 0.2$ & $15.4 \pm 0.2$ & 2.10 & 1640 \\
H & 1 & statistic & $0.39 \pm 0.03$ & $0.003 \pm 0.008$ & 69.4 & 29.3 & 45.8 & 14.0 & $13.3 \pm 0.1$ & $14.7 \pm 0.2$ & 0.15 & 22232 \\
T & 2 & statistic & $0.42 \pm 0.02$ & $-0.05 \pm 0.02$ & 76.7 & 21.9 & 37.3 & 12.8 & $13.7 \pm 0.8$ & $14.7 \pm 0.1$ & 0.90 & 808 \\
P & 2 & statistic & $0.45 \pm 0.02$ & $-0.09 \pm 0.02$ & 56.0 & 42.5 & 46.1 & 14.0 & $14.1 \pm 0.1$ & $15.3 \pm 0.1$ & 0.76 & 1667 \\
B & 2 & statistic & $0.44 \pm 0.03$ & $-0.10 \pm 0.02$ & 47.2 & 51.0 & 55.7 & 15.1 & $14.1 \pm 0.1$ & $16.8 \pm 0.2$ & 0.63 & 851 \\
\hline
\end{tabular}

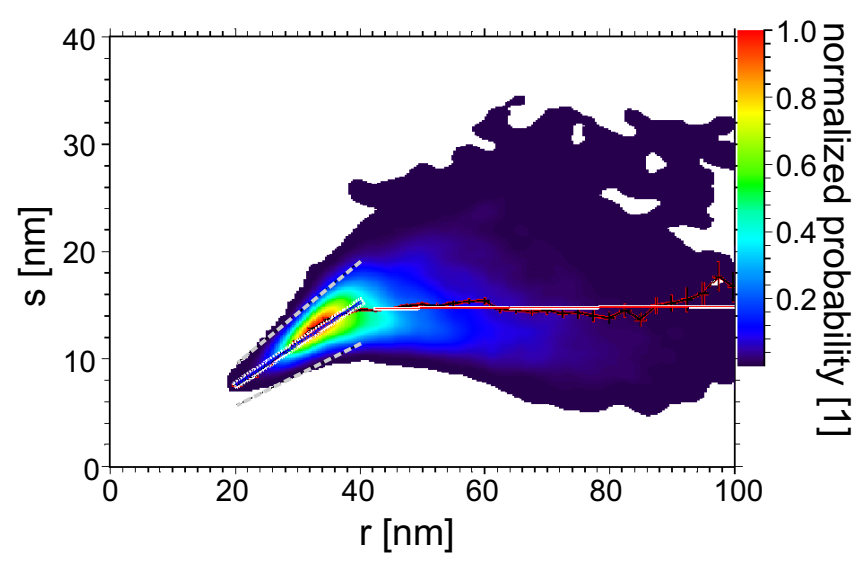

Fig. 1. 2-dimensional probability distribution for mean particle size $(r)$ and distribution width $(s)$ throughout the NLC layer sampled with $150 \mathrm{~m}$ range resolution (method 1 ). The black-red curve indicates the mean distribution width for $2 \mathrm{~nm}$ particle size bins, the errorbars denote the $1-\sigma$ variability. The white-blue line is a errorweighted fit to the data for mean particle sizes below $40 \mathrm{~nm}$. The dashed lines indicate the range for the slope $\pm 25 \%$. The red-white line indicates an fit to the data for $r>40 \mathrm{~nm}$.

The resulting slopes are $S_{<40 \mathrm{~nm}}=0.39 \pm 0.03$ for $r<40 \mathrm{~nm}$ and $S_{\geq 40 \mathrm{~nm}}=0.003 \pm 0.008$ for $r \geq 40 \mathrm{~nm}$. We observe a strong dependence of $s$ and $r$ for mean sizes below about $40 \mathrm{~nm}$, while the mean distribution width is constant at about $s=(14.7 \pm 1.5) \mathrm{nm}$ for particles from 40 to $100 \mathrm{~nm}$.

We have investigated the $s / r$ behavior for different parts of the layer (method 2), as it is shown by observations and model simulations that there is a strong vertical dependence of the particle properties (e.g. von Savigny et al., 2005; Hervig et al., 2009; Baumgarten and Fiedler, 2008). The re- sults are shown in Fig. 2. The mean vertical extent of the NLC top, peak and bottom parts are $0.92 \mathrm{~km}, 0.76 \mathrm{~km}$ and $0.65 \mathrm{~km}$, respectively. For all layer parts we find a strong correlation of $s$ and $r$ for mean sizes up to $40 \mathrm{~nm}$. The slope varies only slightly throughout the layer from 0.41 to 0.44 for the cloud top and bottom respectively. At the mean size of about $r=40 \mathrm{~nm}$ the relationship between $s$ and $r$ changes and we find a decrease of the mean distribution width with increasing $r$. To estimate how well the mean slope describes the whole dataset we have calculated the fraction of particle soundings that deviate by more than $25 \%$ from $s / r=S_{<40 \mathrm{~nm}}$. We find that $79 \%$ (top), $63 \%$ (peak) and $51 \%$ (bottom) of all particle soundings are compatible with the mean slope $\left(S_{<40 \mathrm{~nm}}\right)$. Deviations from the mean slope are mostly caused by larger particles located below the mean slope.

We have investigated the vertical mean particle properties by averaging the signal over the full altitude range (method 3). In Fig. 3 the vertical extent of the sounding volume is about 3-10 times larger than in Fig. 1 and includes the vertical gradient of the mean particle sizes (c.f. Fig. 6). Nevertheless we also find for the vertically integrated signal that about $65 \%$ of the observations are compatible with the mean slope of $S_{<40 \mathrm{~nm}}=0.42 \pm 0.02$. The results for the different analysis methods applied to the complete set of observations are summarized in Table 1. We apply the analysis to two different cloud classes, "all" and "statistic", where the latter only includes clouds with $\beta_{\max }>4 \times 10^{-10} \mathrm{~m}^{-1} \mathrm{sr}^{-1}$. While clouds with $\beta_{\max } \leq 4 \times 10^{-10} \mathrm{~m}^{-1} \mathrm{sr}^{-1}$ could bias the trend analysis due to year-to-year changes in the instrument sensitivity the particle properties are still reliable (Fiedler et al., 2003). For the highest resolution the signal quality allows calculating particle sizes for particles larger than $20 \mathrm{~nm}$, while the small particle size limit is $14-16 \mathrm{~nm}$ for the peak 
Table 2. Particle properties and slope $(S)$ of the fit for different cloud classes and analysis methods. I: Vertical integral, H: Highest resolution, T: NLC top, P: NLC peak, B: NLC bottom. The probability of finding particle soundings close to the fitted slope $( \pm 25 \%)$ is listed in column onfit. The probability for finding particle ensembles where the width is smaller than expected from the fit is listed in column below. Further explanations are given in caption of Table 1.

\begin{tabular}{|c|c|c|c|c|c|c|c|c|c|c|c|c|}
\hline Тур & Method & Cloud & $\begin{array}{c}S_{<40 \mathrm{~nm}} \\
{[1]}\end{array}$ & $\begin{array}{c}S_{\geq 40 \mathrm{~nm}} \\
{[1]}\end{array}$ & $\begin{array}{l}\text { onfit } \\
{[\%]}\end{array}$ & $\begin{array}{c}\text { below } \\
{[\%]}\end{array}$ & $\begin{array}{c}\langle r\rangle \\
{[\mathrm{nm}]}\end{array}$ & $\begin{array}{c}\langle s\rangle \\
{[\mathrm{nm}]}\end{array}$ & $\begin{array}{c}s_{35 \mathrm{~nm}} \\
{[\mathrm{~nm}]}\end{array}$ & $\begin{array}{c}s_{50 \mathrm{~nm}} \\
{[\mathrm{~nm}]}\end{array}$ & $\begin{array}{c}\Delta z \\
{[\mathrm{~km}]}\end{array}$ & Number \\
\hline I & 3 & faint & $0.41 \pm 0.02$ & $1 \pm 0.07$ & 82.5 & 16.7 & 6.1 & 12.5 & 13.9 & 14.2 & 2.35 & 59 \\
\hline I & 3 & weak & $0.41 \pm 0.03$ & $0.01 \pm 0.05$ & 77.5 & 21.2 & 36.9 & 12.5 & 13.8 & 13.8 & 2.23 & \\
\hline I & 3 & medium & $0.45 \pm 0.03$ & $-0.02 \pm 0.03$ & 66.3 & 32.7 & 40.3 & 13.4 & 14.4 & 14.9 & 2.21 & \\
\hline I & 3 & strong & $0.44 \pm 0.04$ & $-0.15 \pm 0.02$ & 52.9 & 41.9 & 49.1 & 15.1 & 15.0 & 16.0 & 1.97 & 94 \\
\hline $\mathrm{H}$ & 1 & fain & $0.39 \pm 0.03$ & $0.08 \pm 0.2$ & 94.2 & 5.8 & 36.3 & 13.5 & 13.6 & 15.7 & 0.15 & 88 \\
\hline $\mathrm{H}$ & 1 & weak & 3 & $-0.031=$ & 89.3 & 10.3 & 38.7 & 13.4 & 13.3 & 8 & 0.15 & 2079 \\
\hline $\mathrm{H}$ & 1 & medium & $0.39 \pm 0.03$ & $0.004 \pm 0.01$ & 81.4 & 17.9 & 40.5 & 13.6 & 13.2 & 14.8 & 0.15 & 6057 \\
\hline $\mathrm{H}$ & 1 & strong & $0.40 \pm 0.03$ & $-0.001 \pm 0.08$ & 59.5 & 39.4 & 49.1 & 14.3 & 13.3 & 14.7 & 0.15 & 14096 \\
\hline $\mathrm{T}$ & 2 & $\mathrm{fa}$ & 0.3 & 0 & 84.2 & 12.3 & 37.4 & 12.7 & 14.0 & 17.0 & 1.25 & 57 \\
\hline $\mathrm{T}$ & 2 & weak & $0.43 \pm$ & $0.11 \pm$ & 88.3 & 11.7 & 36.8 & 13.1 & 13.9 & 17.5 & 0.98 & 11 \\
\hline $\mathrm{T}$ & 0 & medium & $0.45 \pm 0.02$ & $-0.02 \pm 0.03$ & 74.9 & 25.1 & 36.6 & 12.6 & 14.0 & 14.8 & 1.02 & 43 \\
\hline $\mathrm{T}$ & 2 & strong & $0.44 \pm 0.02$ & $--0.03 \pm 0.03$ & 70.7 & 28.4 & 37.8 & 12.9 & 13.8 & 13.7 & 0.82 & 4 \\
\hline $\mathrm{P}$ & 2 & $f_{a}$ & $0.41 \pm$ & $-0.00 \pm 0.07$ & 84.7 & 14.2 & 36.3 & 12.8 & 13.7 & 16.1 & 0.81 & 1 \\
\hline $\mathrm{P}$ & 2 & weak & $0.40 \pm 0.03$ & $-0.02 \pm 0.04$ & 79.3 & 19.5 & 37.3 & 12.4 & 13.4 & 14.0 & 0.78 & 8 \\
\hline $\mathrm{P}$ & 2 & medium & $0.42 \pm 0.03$ & $-0.10 \pm 0.03$ & 68.1 & 29.4 & 41.6 & 13.8 & 13.9 & 14.9 & 0.77 & 524 \\
\hline $\mathrm{P}$ & 2 & strong & $0.42 \pm 0.04$ & $-0.10 \pm 0.02$ & 49.2 & 46.4 & 52.5 & 14.8 & 15.0 & 16.0 & 0.74 & 815 \\
\hline B & 2 & faint & 0. & $4-$ & 76.6 & 23.4 & 42.3 & 14.1 & 14.0 & 16.0 & 0.95 & 7 \\
\hline B & 2 & jeak & $0.46 \pm 0$ & $7-$ & 65.8 & 33.3 & 42.8 & 13.9 & 14.1 & 16.0 & .82 & 11 \\
\hline B & 2 & medium & $0.44 \pm 0.02$ & $-0.10 \pm 0.02$ & 55.9 & 43.7 & 47.0 & 14.2 & 13.7 & 15.4 & 0.73 & 245 \\
\hline B & 2 & strong & $0.49 \pm 0.04$ & $-0.20 \pm 0.01$ & 33.1 & 65.5 & 62.9 & 15.7 & 14.6 & 18.0 & 0.54 & 495 \\
\hline
\end{tabular}

or the integrated layer. Comparing Fig. 1 to Fig. 3 shows that the methods 1 and 3 - both calculating the average relation between $s$ and $r$ for the whole NLC observations - produce different results. For example the slope $S_{\geq 40 \mathrm{~nm}}$ is zero for method 1 but $S_{\geq 40 \mathrm{~nm}}=-0.08 \pm 0.02$ for method 3 similar to the slope at the cloud bottom. One reason is that for method 3 particle properties at the bottom of the cloud have a higher weight as for method 1 . For method 1 all particle properties throughout the layer have the same weight, independent of the particle size. It should be noted that the vertical weighting function depends on the instrument (wavelength) used to derive particle properties (e.g. Hervig et al., 2009). Further discussion of this observation will be given below.

Additionally we have performed the analysis separately for different NLC brightness classes (e.g. Fiedler et al., 2003). We observe a similar slope for all cloud classes and do not find a significant difference for weak to strong clouds. The slope depends slightly on the analysis method where the smallest sounding volume (method 1: $475 \mathrm{~m}$ FWHM smoothing length) shows the lowest slope, about $10 \%$ smaller than the slope of the vertically integrated layer. From weak to strong clouds the number of particle soundings that follow the mean slope varies from $89 \%$ for weak clouds to only $33 \%$ for strong clouds at the NLC bottom. The results for the different analysis methods and cloud classes are com- piled in Table 2. The distribution widths for two fixed mean particle sizes $(35 \mathrm{~nm}$ and $50 \mathrm{~nm}$ ) are explicitly listed in the table. The widths for $35 \mathrm{~nm}$ mean sizes for analysis methods 2 and 3 are usually larger than that of method 1, which uses the highest vertical resolution and hence the smallest sounding volume.

\section{Model results and discussion}

We have analyzed CARMA simulations for 792 different combinations of temperature, $\mathrm{H}_{2} \mathrm{O}$ and eddy diffusion (turbulence) to study the effect of background variability on the NLC particle properties. These are the same model runs as previously described and discussed by Rapp et al. (2007) in the context of modeling color ratios observed by lidar. The key questions to be addressed with the help of these model runs were:

(1) What is the cause for the increase of distribution width with increasing particle size?

(2) What parameters do determine the slope of this increase? (3) Why does the distribution width stop growing at about $40 \mathrm{~nm}$ ? 

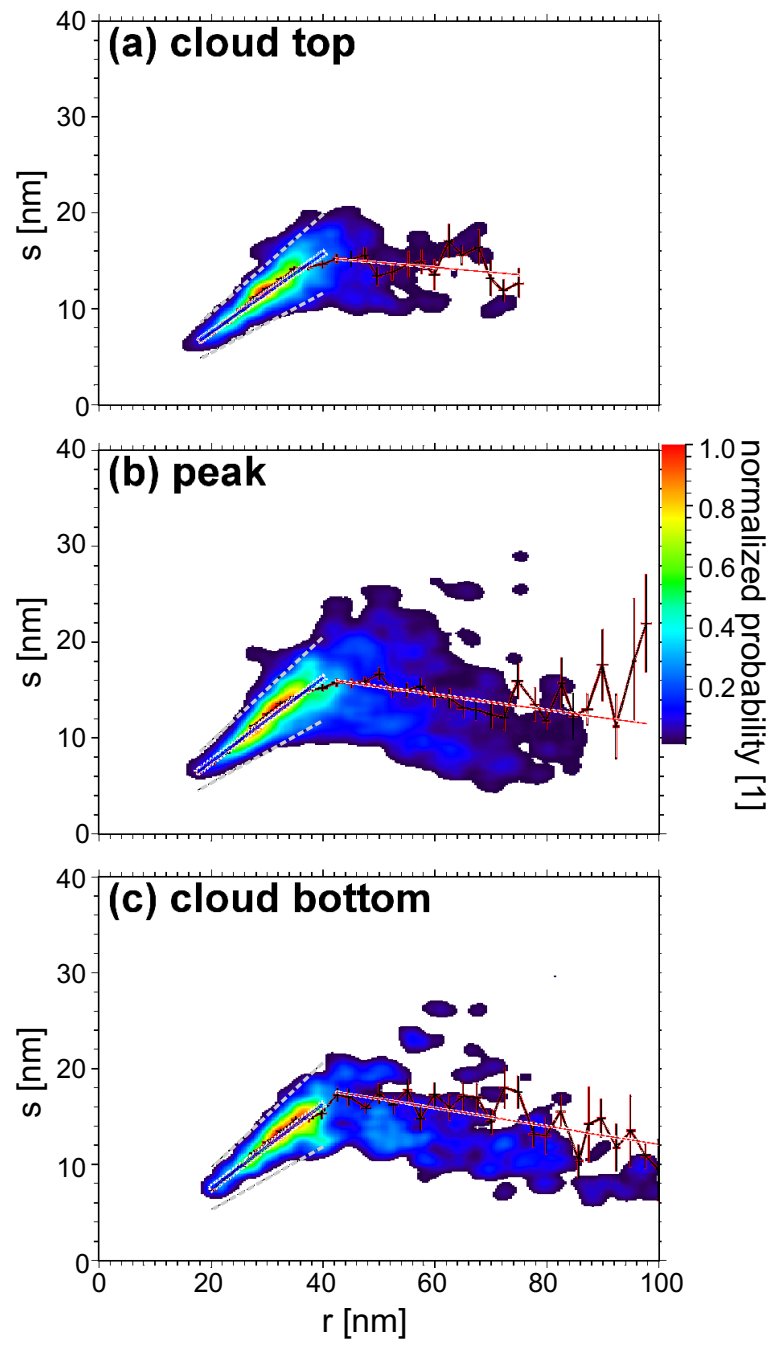

Fig. 2. Same as Fig. 1 but resolved for different altitude regions throughout the NLC layer (method 2). Panels a to c show the cloud properties from top to bottom of the layer.

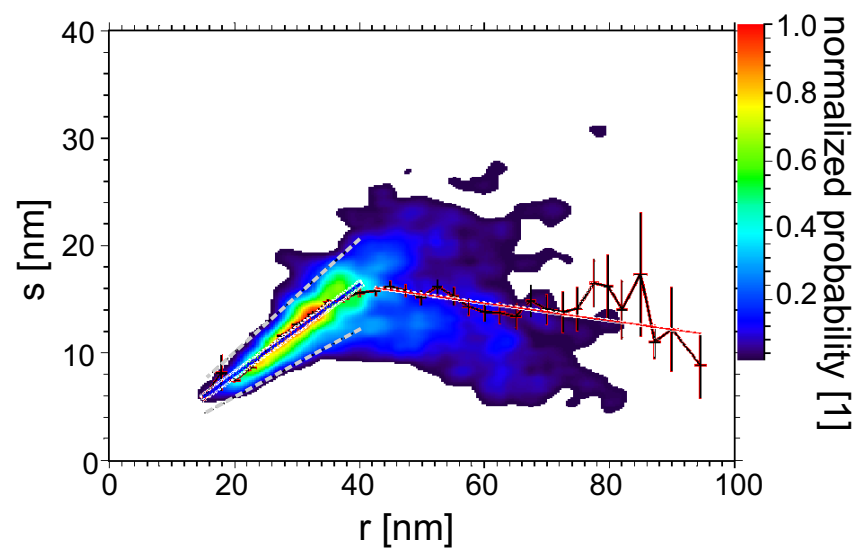

Fig. 3. Mean and width of the size distribution of the signal vertically integrated over the entire NLC layer (method 3). Further explanations see Fig. 1.

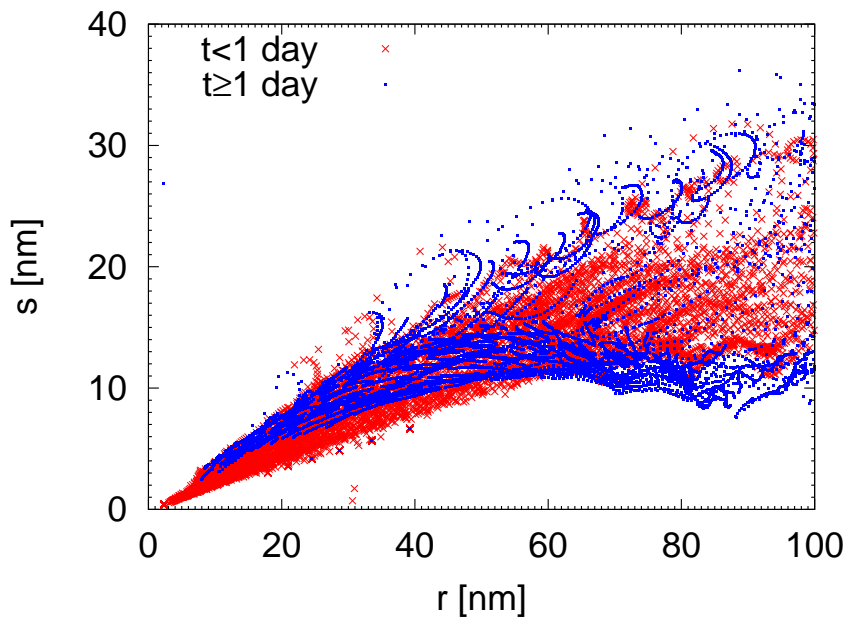

Fig. 4. Mean size and width of the particle size distribution at the peak of the layer for several CARMA simulations with varying temperature (reference $\pm 10 \mathrm{~K}$ ) and $\mathrm{H}_{2} \mathrm{O}$ (reference $\times 0.3-3$ ) background conditions. The red symbols show results from the first $24 \mathrm{~h}$ of simulation, while the blue ones show results from $24-48 \mathrm{~h}$.

(4) What process does cause a decrease of distribution width for large particles?

In Fig. 4 we show the model results for varying temperature and $\mathrm{H}_{2} \mathrm{O}$ but the turbulent eddy diffusion profile of the reference case, discussed in detail in Rapp and Thomas (2006). We observe that the model shows a correlation of mean and width, similar to the lidar observations. In the model the distribution width increases until the mean particle size has reached about $60 \mathrm{~nm}$. Furthermore we observe that the distribution width decreases for larger particles for "mature" clouds i.e., after more than one day of model simulation. This result is compatible to the lidar observations where we find the deviations from the correlation of width and mean size in the lower part of the layer. Here we expect to find the oldest ("mature") particles. Figure 4 also shows that for smaller particles $(r<40 \mathrm{~nm})$ the distribution width of the ensemble for clouds older than one day is slightly larger than that during the first day.

In Fig. 5 we show the mean model results for varying turbulence strength. The eddy diffusion profile of the model reference case was multiplied by factors of 0.1 and 10, respectively. This set of parameters was based on observations of energy dissipation rates that varied about one order of magnitude from the mean (Lübken et al., 2002). It is noteworthy that the turbulence occurrence rate reaches about $90 \%$ at $88 \mathrm{~km}$ and is tightly connected to the mesopause altitude (Rapp and Lübken, 2003, their Fig. 7). Even in the altitude range of $82 \mathrm{~km}$ to $86 \mathrm{~km}$ the turbulence occurrence rate is about $20 \%$ to $50 \%$. We expect that eddy diffusion of less than 0.1 times the mean (i.e. absence of turbulence) is not applicable for NLC growth. Otherwise turbulence must be virtually absent throughout the whole time needed to grow NLC particles from a small nucleus till they can be detected by lidar. 


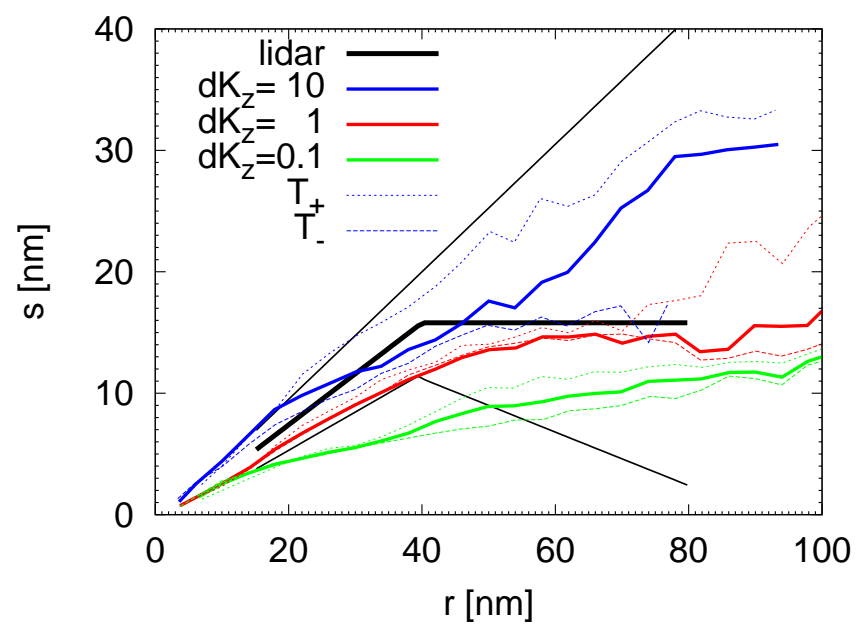

Fig. 5. Mean size and width of the particle size distribution at the peak of the layer for several CARMA simulations with varying background conditions. Solid red, green and blue lines indicate results for varying turbulence for the reference, the reduced and the enhanced case. Dashed and dotted lines indicate $10 \mathrm{~K}$ higher and $10 \mathrm{~K}$ lower temperatures, respectively. The thick black line depicts the size-binned mean distribution width as shown by the fit lines in Fig. 1. Thin black lines indicate the range of observations of $s$ and $r$.

We observe that elevated turbulence also increases the slope of the curve. Furthermore the turbulence strength defines the maximum distribution width reached. For example the reference case asymptotically approaches a width of about $15 \mathrm{~nm}$, similar to the width observed by the lidar. For mean particle sizes larger than $40 \mathrm{~nm}$ the cases with enhanced or reduced turbulence are larger or smaller than the mean width observed by the lidar. For smaller particle sizes the case with enhanced turbulence on the other hand agrees quite well with the observations. The observations with the largest distribution width compare well to the model results with the 10 times enhanced turbulence, but occur less often. Similarly the observations with the smallest distribution width agree with those with reduced turbulence. It should be noted that also mixing due to gravity wave breaking might increase the distribution width but has not been treated in NLC models yet (e.g. Liu and Gardner, 2004). As wave mixing is not included in the model, the eddy diffusion of the reference case might underestimate the broadening of the size distribution due to vertical mixing.

In Fig. 6 we show the eddy diffusion profile and the idealized vertical structure of the particle size. We have extended the lidar observations of the particle size above $85 \mathrm{~km}$ by recent measurements of particle sizes in PMSE (Polar Mesosphere Summer Echos) by radar. Further discussion about the comparison of radar and lidar results can be found in $(\mathrm{Li}$ et al., 2010). Due to the combination of strong eddy diffusion and a gradient in the particle size, turbulent mixing can increase the particle distribution width. The altitude depen-

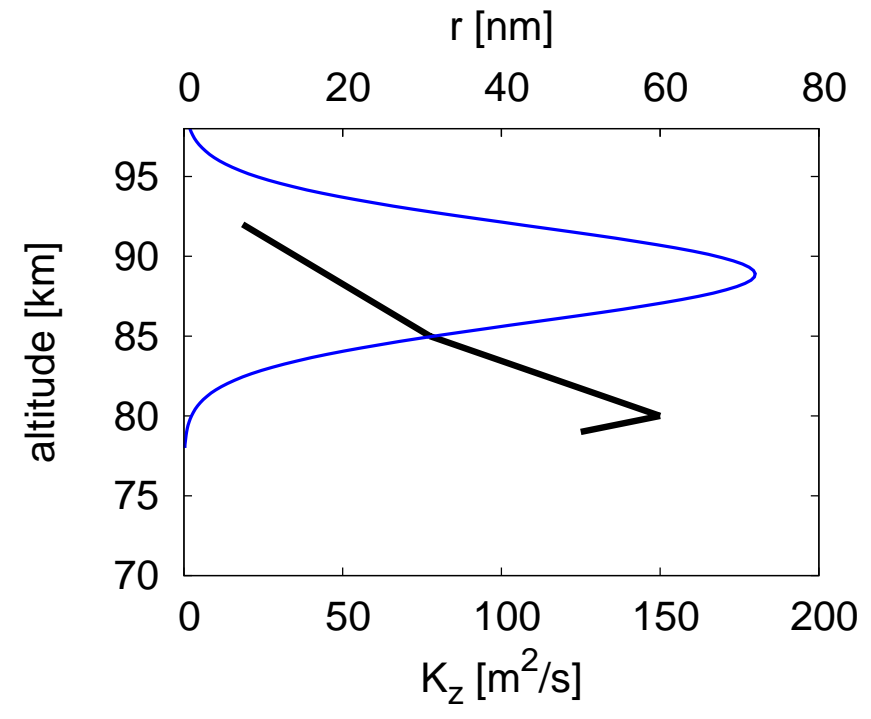

Fig. 6. NLC particle size and eddy diffusion profile for the reference case (blue, lower scale). The profile was derived by fitting a Gaussian to the data published in Lübken (1997). Thick black line depicts an idealized altitude profile of the mean particle size combined from data used in this paper (below $85 \mathrm{~km}$ ), and from radar measurements (above $85 \mathrm{~km}$ ) (Li et al., 2010).

dence of eddy diffusion and particle size also shows a potential mechanism for an upper limit of the mean distribution width. As the turbulence strength decreases below $88 \mathrm{~km}$ but the particle size increases, we can expect a decrease in the broadening of the distribution due to turbulence. For example the mean particle size of $r=40 \mathrm{~nm}$ is reached at an altitude of about $84 \mathrm{~km}$, where the eddy diffusion is only $\sim 25 \%$ of the peak value.

Varying turbulence might also be responsible for the different slopes observed for $r>40 \mathrm{~nm}$ in Fig. 1 to Fig. 3. We observe that method 1 shows a zero slope for $r>40 \mathrm{~nm}$ while methods 2 and 3 resulted in negative slopes throughout the layer. When looking at the size distribution with the highest vertical resolution, we find a larger fraction of clouds with large particles and high distribution widths. This results in a zero slope for $r>40 \mathrm{~nm}$ at the highest resolution (method 1) while the two other methods show a negative slope for $r>40 \mathrm{~nm}$. From the model calculations we realize that distribution widths larger than about $s=20 \mathrm{~nm}$ originate from elevated turbulence. It was previously observed that elevated turbulence can occur in narrow layers $(\Delta z \ll 1 \mathrm{~km}$, Strelnikov et al., 2003). Such narrow layers might be visible in the high resolution data but suppressed when averaging the data vertically (methods 2 and 3 ). The analysis of different cloud classes (Table 2) shows that for methods 2 and 3 the slope is typically negative for strong clouds and for medium clouds at the cloud peak and bottom. So for those cloud classes we observe less clouds with large particles and a wide distribution but more clouds with large particles and a narrow distribution. This might indicate that 
large particles in medium or strong clouds occur more often with reduced turbulence. On the other hand we often observe a decrease of distribution width for particle sizes larger than $40 \mathrm{~nm}$, especially at the lower part of the cloud. This decrease requires another mechanism, as turbulent or wave mixing will increase the distribution width throughout the NLC layer. However, at the bottom of the layer this behavior might change. One reason could be the changing vertical gradient in the mean particle size, causing eddy diffusion to mix volumes of comparable particle sizes. Even more, particles could be mixed downward at the cloud bottom, without upward mixing from below, since particles falling below the cloud bottom will sublimate. Another possible mechanism for decreasing the distribution width is that vertical winds, introduced by gravity waves, can separate small particles from the larger ones. Potentially, redistribution of water to lower altitudes by freeze-drying also might shift the nucleation to lower altitudes where turbulence is less active.

\section{Conclusions}

We have presented an extensive set of lidar observations of NLC particle properties and analyzed the dataset regarding the mean size $(r)$ and distribution width $(s)$. We observe a robust correlation of $s$ and $r$ for mean sizes up to $40 \mathrm{~nm}$. This relation is independent of the analysis method and can be found throughout the NLC layer. The distribution width $s$ can be approximated from the mean particle size by $s=0.4 \times r$. Allowing an accuracy of $\pm 25 \%, 70 \%$ of the lidar observations, analyzed with the highest possible vertical resolution, are compatible with this simple relationship. The approximation becomes even better if in addition a constant distribution width of $s \approx 15 \mathrm{~nm}$ is used for $r \geq 40 \mathrm{~nm}$.

A microphysical model of NLC (CARMA) can reproduce the observed correlation for smaller particles. We find that the slope is defined by the strength of vertical mixing due to eddy diffusion. A smaller fraction of observations shows a combination of $s$ and $r$ that can be explained by 10 times increased eddy diffusion. The model also reproduces that the distribution width for larger particles becomes constant. This constant distribution width could be caused by the fact that we observe larger particles in the lower part of the NLC layer. At altitudes where the mean particle size is above $40 \mathrm{~nm}$, the diffusion coefficient is at most $25 \%$ of the peak value of eddy diffusion.

Our investigation shows that the observed dependence of $s$ on $r$ is a valuable tool to adjust the microphysical processes in modeling of NLC, primarily the eddy diffusion or wave mixing. The observed approximation of distribution width as function of particle size is of importance for other instruments that can not directly measure the distribution width and the particle size.
Acknowledgements. We gratefully acknowledge the support of the ALOMAR staff and helping to accumulate the extensive data set of NLC observations. The observations were supported by a huge number of voluntary lidar operators. The most recent summer students were Carsten Baumann and Mandy Bethkenhagen. This project received research funding from the European Community's 6th Framework Program under the project "ALOMAR eARI" (RITA-CT-2003-506208)

Edited by: S. Buehler

\section{References}

Bailey, S. M., Thomas, G. E., Rusch, D. W., Merkel, A. W., Jeppesen, C. D., Carstens, J. N., Randall, C. E., McClintock, W. E., and Russell, J. M.: Phase functions of polar mesospheric cloud ice as observed by the CIPS instrument on the AIM satellite, J. Atmos. Solar-Terr. Phys., 71, 373-380, doi:10.1016/j.jastp.2008.09.039, 2009.

Baumgarten, G. and Fiedler, J.: Vertical structure of particle properties and water content in noctilucent clouds, Geophys. Res. Lett., 35, L10811, doi:10.1029/2007GL033084, 2008.

Baumgarten, G., Lübken, F.-J., and Fricke, K. H.: First observation of one noctilucent cloud by a twin lidar in two different directions, Ann. Geophys., 20, 1863-1868, doi:10.5194/angeo20-1863-2002, 2002.

Baumgarten, G., Fiedler, J., and von Cossart, G.: The size of noctilucent cloud particles above ALOMAR (69N,16E): Optical modeling and method description, 40, 772-784, doi:10.1016/j. asr.2007.01.018, 2007.

Baumgarten, G., Fiedler, J., Lübken, F.-J., and von Cossart, G.: Particle properties and water content of noctilucent clouds and their interannual variation, J. Geophys. Res., 113, D06203, doi: 10.1029/2007JD008884, 2008.

Berger, U. and von Zahn, U.: Icy particles in the summer mesopause region: Three-dimensional modeling of their environment and two-dimensional modeling of their transport, J. Geophys. Res., 107, 1366, doi:10.1029/2001JA000316, 2002.

Fiedler, J., von Cossart, G., and Baumgarten, G.: Noctilucent clouds above ALOMAR between 1997 and 2001: Occurrence and properties, J. Geophys. Res., 108, 8453, doi:10.1029/2002JD002419, 2003.

Fiedler, J., Baumgarten, G., and Lübken, F.-J.: NLC observations during one solar cycle above ALOMAR, J. Atmos. Solar-Terr. Phys., 71, 424-433, doi:10.1016/j.jastp.2008.11.010, 2009.

Fritts, D. C., Isler, J. R., Thomas, G. E., and Andreassen, Ø.: Wave breaking signatures in noctilucent clouds, Geophys. Res. Lett., 20, 2039-2042, 1993.

Gadsden, M. and Schröder, W.: Noctilucent clouds, SpringerVerlag, New York, USA, ISBN: 0387506853, 1989.

Hervig, M., Thompson, R. E., McHugh, M., Gordley, L. L., Russel III, J. M., and Summers, M. E.: First confirmation that water ice is the primary component of polar mesospheric clouds, Geophys. Res. Lett., 28, 971-974, doi:10.1029/2000GL012104, 2001.

Hervig, M. E., Gordley, L. L., Stevens, M. H., Russell, J. M., Bailey, S. M., and Baumgarten, G.: Interpretation of SOFIE PMC measurements: Cloud identification and derivation of mass den- 
sity, particle shape, and particle size, J. Atmos. Solar-Terr. Phys., 71, 316-330, doi:10.1016/j.jastp.2008.07.009, 2009.

Jensen, E. J. and Thomas, G. E.: Numerical simulations of the effects of gravity waves on noctilucent clouds, J. Geophys. Res., 99, 3421-3430, doi:10.1029/93JD01736, 1994.

Li, Q., Rapp, M., Röttger, J., Latteck, R., Zecha, M., Strelnikova, I., Baumgarten, G., Hervig, M., Hall, C., and Tsutsumi, M.: Microphysical parameters of mesospheric ice clouds derived from calibrated observations of polar mesosphere summer echoes at Bragg wavelengths of $2.8 \mathrm{~m}$ and $30 \mathrm{~cm}$, J. Geophys. Res., 115, D00I13, doi:10.1029/2009JD012271, 2010.

Liu, A. Z. and Gardner, C. S.: Vertical dynamical transport of mesospheric constituents by dissipating gravity waves, J. Atmos. Sol.Terr. Phys., 66, 267-275, doi:10.1016/j.jastp.2003.11.002, 2004.

Lübken, F.-J.: Seasonal variation of turbulent energy dissipation rates at high latitudes as determined by in situ measurements of neutral density fluctuations, J. Geophys. Res., 102, 1344113456, doi:10.1029/97JD00853, 1997.

Lübken, F.-J., Rapp, M., and Hoffmann, P.: Neutral air turbulence and temperatures in the vicinity of polar mesosphere summer echoes, J. Geophys. Res., 107, 4273, doi:10.1029/ 2001JD000915, 2002.

Lübken, F.-J.: Thermal structure of the arctic summer mesosphere, J. Geophys. Res., 104, 9135-9149, doi:10.1029/1999JD900076, 1999.

Rapp, M. and Lübken, F.-J.: On the nature of PMSE: Electron diffusion in the vicinity of charged particles revisited, J. Geophys. Res., 108, 8437, doi:10.1029/2002JD002857, 2003.

Rapp, M. and Thomas, G. E.: Modeling the microphysics of mesospheric ice particles: Assessment of current capabilities and basic sensitivities, J. Atmos. Solar-Terr. Phys., 68, 715-744, doi: 10.1016/j.jastp.2005.10.015, 2006.

Rapp, M., Lübken, F.-J., Müllemann, A., Thomas, G., and Jensen, E.: Small scale temperature variations in the vicinity of NLC: Experimental and model results, J. Geophys. Res., 107, 4392, doi:10.1029/2001JD001241, 2002.

Rapp, M., Thomas, G. E., and Baumgarten, G.: Spectral properties of mesospheric ice clouds: Evidence non-spherical particles, J. Geophys. Res., 112, 3211, doi:10.1029/2006JD007322, 2007.
Robert, C. E., von Savigny, C., Burrows, J. P., and Baumgarten, G.: Climatology of noctilucent cloud radii and occurrence frequency using SCIAMACHY, J. Atmos. Solar-Terr. Phys., 71, 408-423, doi:10.1016/j.jastp.2008.10.015, 2009.

Seele, C. and Hartogh, P.: Water vapor of the polar middle atmosphere: Annual variation and summer mesosphere conditions as observed by ground-based microwave spectroscopy, Geophys. Res. Lett., 26, 1517-1520, doi:10.1029/1999GL900315, 1999.

Strelnikov, B., Rapp, M., and Lübken, F.-J.: A new technique for the analysis of neutral air density fluctuations measured in situ in the middle atmosphere, Geophys. Res. Lett., 30, 2052, doi: 10.1029/2003GL018271, 2003.

Thomas, G. E.: Solar Mesosphere Explorer measurements of polar mesospheric clouds (noctilucent clouds), J. Atmos. Terr. Phys., 46, 819-824, 1984.

Toon, O. B., Turco, R. P., Hamill, P., Kiang, C. S., and Whitten, R. C.: A One-Dimensional Model Describing Aerosol Formation and Evolution in the Stratosphere: II. Sensitivity Studies and Comparison with Observations., J. Atmos. Sci., 36, 718-736, 1979.

Turco, R. P., Hamill, P., Toon, O. B., Whitten, R. C., and Kiang, C. S.: A One-Dimensional Model Describing Aerosol Formation and Evolution in the Stratosphere: I. Physical Processes and Mathematical Analogs., J. Atmos. Sci., 36, 699-717, 1979.

Turco, R. P., Toon, O. B., Whitten, R. C., Keesee, R. G., and Hollenbach, D.: Noctilucent Clouds: Simulation Studies of their Genesis, Properties and Global Influences, Planet. Space Sci., 30, 1147-1181, 1982.

von Savigny, C., Petelina, S. V., Karlsson, B., Llewellyn, E. J., Degenstein, D. A., Lloyd, N. D., and Burrows, J. P.: Vertical variation of NLC particle sizes retrieved from Odin/OSIRIS limb scattering observations, Geophys. Res. Lett., 32, L07806, doi: 10.1029/2004GL021982, 2005.

Witt, G.: Height, structure and displacements of noctilucent clouds, Tellus, 14, 1-18, 1962. 